\title{
FACTORIZATION AND BOUNDED APPROXIMATE IDENTITIES FOR A CLASS OF CONVOLUTION BANACH ALGEBRAS
}

\author{
by S. I. OUZOMGI
}

(Received 10 July, 1985)

An algebra $A$ factors if, for each $a \in A$, there exist $b, c \in A$ with $a=b c$. A bounded approximate identity for a Banach algebra $A$ is a net $\left(e_{\alpha}\right) \subset A$ such that $a e_{\alpha} \rightarrow a$ and $e_{\alpha} a \rightarrow a$ for each $a \in A$ and such that sup $\left\|e_{\alpha}\right\|<\infty$. It is well known $[2,11.10]$ that if $A$ has a bounded approximate identity, then $A$ factors. But a Banach algebra may factor even if it does not have a bounded approximate identity: an example which is non-commutative and separable, and an example which is commutative and nonseparable, are given in $[3, \S 22]$. However, we do not know an example of a commutative, separable Banach algebra which factors, but which does not have a bounded approximate identity. See [4] for related work.

In this note, we show that, for a certain class of commutative, separable Banach algebras, an algebra factors if and only if it has a bounded approximate identity.

A real-valued function $\omega$ defined on $\mathbb{R}^{+}$is a weight function if $\omega$ is Lebesgue measurable, if $\omega(t)>0\left(t \in \mathbb{R}^{+}\right)$, and if

$$
\omega(s+t) \leqslant \omega(s) \omega(t) \quad\left(s, t \in \mathbb{R}^{+}\right) .
$$

Let $\omega$ be a weight function on $\mathbb{R}^{+}$. We denote by $L^{1}(\omega)$ the set of complex-valued, measurable functions on $\mathbb{R}^{+}$such that

$$
\|f\| \equiv \int_{0}^{\infty}|f(t)| \omega(t) d t<\infty .
$$

As usual, we equate functions which are equal almost everywhere. Then $L^{1}(\omega)$ is a Banach space with respect to pointwise addition and scalar multiplication. For $f, g \in L^{1}(\omega)$, we define $f * g$ by setting

$$
(f * g)(t)=\int_{0}^{t} f(t-s) g(s) d s \quad\left(t \in \mathbb{R}^{+}\right) .
$$

Then $f * g$ is finite almost everywhere and defines an element of $L^{1}(\omega)$. With respect to this convolution multiplication, $L^{1}(\omega)$ is a commutative Banach algebra, and clearly $L^{1}(\omega)$ is separable. The algebras $L^{1}(\omega)$ are discussed in [1], for example.

In the theorem below, we write $m$ for Lebesgue measure on $\mathbb{R}^{+}$and supp $f$ for the support of a function $f$. If $A$ is an algebra, then $A^{2}$ denotes the linear span of the set of products of two elements of $A$.

THEOREM. Let $\omega$ be a weight function on $\mathbb{R}^{+}$. Then the following conditions on $\omega$ are Glasgow Math. J. 28 (1986) 211-214. 
equivalent: than 0 ;

(1) there exists $M>0$ such that, for each $\delta>0, m\{t \in[0, \delta]: \omega(t)<M\}$ is greater

(2) $L^{1}(\omega)$ has a bounded approximate identity;

(3) $L^{1}(\omega)$ factors;

(4) $\left[L^{1}(\omega)\right]^{2}=L^{1}(\omega)$.

Proof. (1) $\Rightarrow(2)$. Let $E_{n}=\{t \in(0,1 / n]: \omega(t) \leqslant M\}$. By hypothesis, $m\left(E_{n}\right)>0$. Let $\chi_{n}$ be the characteristic function of $E_{n}$, and let $e_{n}=\chi_{n} / m\left(E_{n}\right)$. Clearly, $\left\|e_{n}\right\| \leqslant M$, and so $\left(e_{n}\right)$ is a bounded sequence in $L^{1}(\omega)$.

A standard argument using the uniform continuity of a continuous function with compact support shows that $\left(e_{n}\right)$ is a bounded approximate identity for $L^{1}(\omega)$.

$(2) \Rightarrow(3)$. This is Cohen's factorization theorem $[2,11.10]$.

(3) $\Rightarrow$ (4). Immediate.

$(4) \Rightarrow(1)$. To obtain a contradiction, suppose that (4) holds but that (1) fails. Define a function $\bar{\omega}$ on $\mathbb{R}^{+}$by setting

$$
\tilde{\omega}(t)=\operatorname{ess} \inf \{\omega(s): 0<s<t\} \quad(t>0) .
$$

Then $\tilde{\omega}$ is measurable on $\mathbb{R}^{+}$, and $\tilde{\omega}(t) \leqslant \omega(t)$ for almost all $t>0$. Take $s, t>0$ and $\varepsilon>0$. Then there are sets $S \subset(0, s)$ and $T \subset(0, t)$ such that $S$ and $T$ have positive measure and such that

$$
\omega\left(s^{\prime}\right) \leqslant \tilde{\omega}(s)+\varepsilon \quad\left(s^{\prime} \in S\right), \quad \omega\left(t^{\prime}\right) \leqslant \tilde{\omega}(t)+\varepsilon \quad\left(t^{\prime} \in T\right) .
$$

Then $S+T$ is a subset of $(0, s+t)$ which has positive measure, and

$$
\omega\left(s^{\prime}+t^{\prime}\right) \leqslant \omega\left(s^{\prime}\right) \omega\left(t^{\prime}\right) \leqslant(\bar{\omega}(s)+\varepsilon)(\bar{\omega}(t)+\varepsilon) \quad\left(s^{\prime} \in S, t^{\prime} \in T\right) .
$$

Thus $\tilde{\omega}(s+t) \leqslant(\tilde{\omega}(s)+\varepsilon)(\tilde{\omega}(t)+\varepsilon)$. This is true for each $\varepsilon>0$, and so $\tilde{\omega}(s+t) \leqslant$ $\tilde{\omega}(s) \bar{\omega}(t)$. Hence $\tilde{\omega}$ is a weight function on $\mathbb{R}^{+}$, because $\bar{\omega}$ is measurable. Further $\bar{\omega}$ is decreasing.

Define a function $\Omega$ on $(0, \infty)$ by

$$
\Omega(\delta)=\sup \left\{\frac{\bar{\omega}(s+t)}{\bar{\omega}(s) \bar{\omega}(t)}: s, t>0, s+t \leqslant \delta\right\} \quad(\delta>0) .
$$

Clearly, $\Omega$ is monotonically increasing on $(0, \infty)$. Since (1) fails, $\tilde{\omega}(t) \rightarrow \infty$ as $t \rightarrow 0+$, and so $\Omega(\delta) \rightarrow 0$ as $\delta \rightarrow 0+$.

For $t>0$, set

$$
S_{t}=\{s \in(0, t): \omega(s) \leqslant 2 \bar{\omega}(t)\} .
$$

Then $S_{t}$ has positive measure, and $\omega(s) \leqslant 2 \tilde{\omega}(s)\left(s \in S_{t}\right)$. We can inductively define a sequence $\left(\delta_{n}\right)$ such that $0<\delta_{n+1}<\delta_{n}$, such that $\sum_{n=1}^{\infty} \Omega\left(\delta_{n}\right)<\infty$, and such that $m\left(A_{n}\right)>0$, where $A_{n}=S_{\delta_{n}} \cap\left(\delta_{n+1}, \delta_{n}\right)$. 
Set

$$
f(t)=\sum_{n=1}^{\infty} \frac{\Omega\left(\delta_{n}\right)}{m\left(A_{n}\right) \omega(t)} \chi_{A_{n}}(t) \quad(t>0)
$$

Then $\int_{0}^{\infty}|f(t)| \omega(t) d t=\sum_{n=1}^{\infty} \Omega\left(\delta_{n}\right)<\infty$, and so $f \in L^{1}(\omega)$.

We shall show that $f \notin\left[L^{1}(\omega)\right]^{2}$. To obtain a contradiction, suppose that $f=\sum_{i=1}^{k} g_{i} * h_{i}$, where $g_{1}, \ldots, g_{k}, h_{1}, \ldots, h_{k} \in L^{1}(\omega)$. Then

$$
f(t) \leqslant \sum_{i=1}^{k} \int_{0}^{t}\left|g_{i}(t-s) h_{i}(s)\right| d s \quad\left(t \in \mathbb{R}^{+}\right) .
$$

Since $\tilde{\omega}(t) \leqslant \omega(t)$ for almost all $t$ and $\omega(t) \leqslant 2 \tilde{\omega}(t)$ for $t \in \operatorname{supp} f$, we have

$$
\Omega\left(\delta_{n}\right)=\int_{A_{n}} f(t) \omega(t) d t \leqslant 2 \Omega\left(\delta_{n}\right) K_{n},
$$

where

$$
K_{n}=\sum_{i=1}^{k} \int_{A_{n}} \int_{0}^{t}\left|g_{i}(t-s) h_{i}(s)\right| \omega(t-s) \omega(s) d s d t .
$$

Thus $K_{n} \geqslant 1 / 2(n \in \mathbb{N})$. However,

$$
\begin{aligned}
\sum_{n=1}^{\infty} K_{n} & \leqslant \sum_{i=1}^{k} \int_{0}^{\infty} \int_{0}^{t}\left|g_{i}(t-s)\right| \omega(t-s)\left|h_{i}(s)\right| \omega(s) d s d t \\
& \leqslant \sum_{i=1}^{k}\left\|g_{i}\right\|\left\|h_{i}\right\|<\infty
\end{aligned}
$$

and so $K_{n} \rightarrow 0$ as $n \rightarrow \infty$. This is the required contradiction.

This completes the proof of the theorem.

REMARK. If $\omega$ is bounded in a neighborhood of 0 , then clearly the conditions of the theorem are satisfied. However, it is easy to give a weight function $\omega$ for which ess $\lim \sup \omega(t)=\infty$, but which satisfies the conditions of the theorem.

In the above proof, we introduced a new weight function $\tilde{\omega}$. This was necessary because there are weight functions $\omega$ for which (1) fails, but which are such that

$$
\inf _{\delta>0} \operatorname{ess} \sup \left\{\frac{\omega(s+t)}{\omega(s) \omega(t)}: s, t>0, s+t \leqslant \delta\right\}>0 .
$$

To exemplify these two remarks, we give one construction.

Let $\left(c_{n}\right),\left(\delta_{n}\right)$ be sequences with $c_{1}=0, c_{n+1}>c_{n}, \delta_{1}=1$, and $0<\delta_{n+1}<\delta_{n}$ for $n \in \mathbb{N}$ and $\delta_{n} \rightarrow 0$ as $n \rightarrow \infty$. Let $\eta_{n}(t)=\left(c_{n+1}-c_{n}\right) t\left(t \in\left[0, \delta_{n}\right]\right)$ and let $\eta_{n}(t)=0\left(t>\delta_{n}\right)$. Then 
$\eta_{n}(s+t) \leqslant \eta_{n}(s)+\eta_{n}(t)$ for $s, t \in \mathbb{R}^{+}$. Let $\eta(t)=\sum \eta_{n}(t)$, and let $\omega(t)=\exp \eta(t)\left(t \in \mathbb{R}^{+}\right)$. Then $\omega$ is a weight function on $\mathbb{R}^{+}$, and $\eta(t)=c_{n+1} t\left(t \in\left(\delta_{n+1}, \delta_{n}\right]\right)$. Suppose further that $\delta_{n+1}<\delta_{n} / n$ and that $c_{n+1}=n / \delta_{n}(n \in \mathbb{N})$. On $\left[\delta_{n} / n, 2 \delta_{n} / n\right], \eta(t) \leqslant 2$, and so $\omega$ satisfies condition (1), above. However, on $\left[\delta_{n} / 2, \delta_{n}\right], \eta(t) \geqslant n / 2$, and so ess $\lim _{t \rightarrow 0+} \sup \omega(t)=\infty$.

Secondly, take $\omega$ as above, choosing $\delta_{n+1}<\delta_{n} / 4$ and $c_{n}=n / \delta_{n}(n \in \mathbb{N})$. Then $\omega(s+t)=\omega(s) \omega(t)$ for $s, t \in\left(\frac{1}{4} \delta_{n}, \frac{1}{2} \delta_{n}\right)$. However, $\eta(t) \geqslant c_{n+1} \delta_{n+1}$ for $t \in\left(0, \delta_{n}\right]$, and so (1) fails.

Acknowledgement. This paper is a part of a Ph.D. thesis submitted to Leeds University. I should like to thank my supervisor H. G. Dales for his constant help and encouragement.

\section{REFERENCES}

1. W. G. Bade and H. G. Dales, Norms and ideals in radical convolution algebras. J. Funct. Anal. 41 (1981), 77-109.

2. F. F. Bonsall and J. Duncan, Complete normed algebras (Springer, 1973).

3. R. S. Doran and J. Wichmann, Approximate identities and factorization in Banach modules, Lecture Notes in Mathematics 768 (Springer, 1979).

4. S. I. Ouzomgi, Factorization and automatic continuity for an algebra of infinitely differentiable functions, J. London Math. Soc. (2) 30 (1984), 265-280.

Department of Mathematics and Computer Science

California State University, Long Beach

Long BEACH, CaLifornia 90840 